\title{
Label de calidad e indicaciones geográficas protegidas como estrategia empresarial: El caso Brandy de Jerez desde una perspectiva de las RR.PP
}

\author{
Marta Pulido \\ Universidad de Cádiz \\ Gloria Jiménez \\ Universidad de Sevilla \\ Carmen Silva \\ Universidad de Cádiz
}

\section{Palabras clave}

Brandy de Jerez, Consejo Regulador, Indicación Geográfica Protegida, Label de calidad, Relaciones Públicas

\section{Resumen}

La Indicación Geográfica Protegida (IGP), como label de calidad, supone una doble perspectiva: un valor añadido a la marca del producto, al tiempo que es una garantía de calidad mínima. Sirve para hacer alusión a productos de la industria agroalimentaria que se identifican por unas características diferentes y propias que se derivan de su procedencia geográfica, añadiendo percepción pública del mismo, controles y normativas estrictas de elaboración y un marco legal específico. En este sentido, las indicaciones geográficas protegidas juegan un importante papel en la gestión empresarial y, desde una óptica comunicacional, un rol fundamental en la gestión de las RR.PP. En este estudio realizamos un estudio del Consejo Regulador de la indicación geográfica protegida Brandy de Jerez a través del estudio de caso combinado con otras técnicas de investigación como son la entrevista, la observación directa y la consulta de fuentes de datos secundarios. Consideramos, pues, a partir de las veinte memorias anuales que el Consejo Regulador de la indicación geográfica protegida Brandy de Jerez emitió desde 1989 hasta 2008, la relación existente entre el label de calidad IGP y la gestión de las RR.PP. de las empresas y productos que quedan bajo el amparo esta protección. 


\title{
Quality label and geographical indications as a business strategy: The case of 'Brandy de Jerez' from a PR perspective
}

\section{Keywords}

Sherry, Regulatory Conceil, 'Indicación Geográfica Protegida', Quality label de calidad, Public Relations

\begin{abstract}
'Indicación Geográfica Protegida' (IGP) as quality label have a double perspective: value to the product brand and quality assurance that it is add to the product. Used to make reference to the food industry products that are identified by different characteristics and own derived from their geographical origin, adding public perception thereof, strict controls and regulations development and a specific legal framework. In this sense, geographical indications play an important role in business management from a communication perspective, a fundamental role in the management of $P R$ in this study conducted a study of the 'Consejo Regulador' of the protected geographical indication 'Brandy de Jerez' (Sherry) through the case study combined with other investigative techniques such as interviews, direct observation and consultation of secondary data sources. We believe, therefore, from the twenty annual reports to the 'Consejo Regulador de la indicación geográfica protegia del Brandy de Jerez' issued from 1989 to 2008, the relationship between the IGP quality label and management of PR companies and products that fall under the umbrella protection.
\end{abstract}

\section{Autoras}

Marta Pulido [marta.pulido@uca.es] es doctora y profesora en la Facultad de Ciencias Sociales y de la Comunicación en la Universidad de Cádiz.

Gloria Jiménez [gloria_jimenez@us.es] es doctora y profesora en la Facultad de Comunicación de la Universidad de Sevilla.

Carmen Silva Robles [carmen.silva@uca.es] es doctoranda en la Facultad de Ciencias Sociales y Comunicación de la Universidad de Cádiz. 


\section{Introducción}

El siguiente texto parte del interés por investigar sobre un fenómeno, la indicación geográfica como label de calidad, y se justifica desde una doble perspectiva: primero desde el valor que supone una indicación geográfica protegida y, en segundo lugar, en relación al crecimiento que, en los últimos años, se encuentran experimentando estas indicaciones en España.

Partimos de que la expresión Indicación geográfica es el término que, de forma genérica y básica, nos sirve para hacer alusión a un fenómeno por el cual una serie de productos de la industria agroalimentaria se identifican por unas características diferentes y propias. Así, el valor que aporta una indicación geográfica protegida supone que no es lo mismo hablar de un vino $\mathrm{x}$, que de «vino de Jerez», por ejemplo. Y es que, los valores que se derivan de su procedencia geográfica, aquellos que indican su procedencia, le proporcionan un valor añadido al producto en varias dimensiones:

Por una parte, un valor que se relaciona con la percepción pública del producto en relación con la confianza que genera su calidad y las expectativas que suponen la asociación a su origen.

Por otra, un valor que se relaciona con un sector productivo sometido a controles y normativas estrictas de elaboración.

En tercer lugar, un valor que es digno de ser reconocido oficialmente, como demuestra el desarrollo, a lo largo de la historia, de un marco legal orientado a su protección jurídica.

En este sentido, las indicaciones geográficas protegidas juegan un importante papel en la gestión de la comunicación del sector vitivinícola.

En relación al aumento de indicaciones geográficas en España en los últimos años, debe destacarse que solo en 2002 el número de indicaciones geográficas «creció en nuestro país de forma espectacular, pasando a superar la centena» (Martínez Ruiz y Jiménez Zarco, 2006:27). De este modo, destacamos que en la actualidad existen en España más de 300 indicaciones geográficas protegidas repartidas entre diferentes sectores del ámbito agroalimentario. Y solo en 2011 casi la mitad de las indicaciones geográficas protegidas en España se concentraban en torno al sector vitivinícola con vinos con Denominación de Origen Protegida (con un 27,96 \% del total de indicaciones geográficas protegidas en España) y vinos con mención Vinos de la Tierra (que representa el 14,47 \%).

\section{Objetivos}

En la actualidad, de manera lógica, nos encontramos con una amplia oferta de productos de todo tipo (agroalimentarios, turísticos, informáticos, mediáticos, etc.). Ocurre que cada producto y categoría buscan y piensan sus diversas es- 
trategias empresariales y comunicativas para dar una información acerca de sí mismos de la manera más eficaz posible. Y, en el caso que nos ocupa, vender, en sentido literal y figurado, más y mejor.

Así, desde el punto de vista de la investigación de mercado sucede que, el contacto directo del producto, en este caso, vitivinícola, con los públicos y su predilección por unas características u otras (calidad, precio...) convierten a la indicación geográfica en una pieza fundamental en el estudio de las necesidades y deseos de los consumidores y las tendencias de compras en los mercados de los sectores que interesan a las empresas.

Desde la organización de empresas y siguiendo la teoría del análisis sistémico, tan empleada en investigación sobre RR.PP., se contempla la vinculación de la indicación geográfica con la comunicación estratégica de la corporación y sus distintos departamentos, posicionándose como un elemento más que trabaja con todas las áreas de la organización, suponiendo un elemento básico y fundamental a la hora de vender el mismo. Y esto es así por su capacidad de vincular la calidad que supone un determinado clima, un sistema de producción, o un terreno específico en la calidad final del producto. Y a veces, también en el precio (aunque no siempre al alza, no siempre a la baja).

Así, el objetivo del que parte este estudio es la demostración de la relevancia que supone una indicación geográfica protegida como label de calidad en cualquier sector y, más específicamente, en el sector vitivinícola, siempre desde una óptica estratégica y comunicativa, no necesariamente publicitaria. Así, y partiendo de este objetivo genérico, nos planteamos demostrar que las indicaciones geográficas tienen, además, un valor añadido para el producto en cuestión, de manera que quedan vinculados: origen geográfico y gestión de la comunicación. Es, pues, la gestión estratégica de la comunicación de un producto agroalimentario (el vino) a través de las características propias e innatas del mismo.

\section{Metodología de investigación}

Este estudio es fruto de una investigación en la que se combinan métodos cualitativos y cuantitativos de recogida de datos para su posterior análisis. En concreto se lleva a cabo un análisis de contenido sumado a un análisis de discurso y un estudio de caso concreto: estudio del Consejo Regulador de la indicación geográfica protegida Brandy de Jerez. Para llevar a cabo la aplicación del estudio de caso se emplean las siguientes técnicas de investigación: estudio de caso combinado, entrevista, observación directa y consulta de fuentes de datos secundarios. Todo ello a partir de las veinte memorias anuales que el Consejo Regulador de la indicación geográfica protegida Brandy de Jerez emitió desde 1989 hasta 2008.

El análisis de contenido es una técnica de investigación que se fundamenta en una descripción cuantitativa, metódica y objetiva del contenido de cualquier co- 
municación (Berelson, 1968). En esta investigación validaremos mediante esta técnica un conjunto de datos, cuantificando una serie de cambios y modificaciones aparecidas en las diversas memorias anuales del Consejo Regulador de la indicación geográfica protegida Brandy de Jerez. Concretamente en las veinte memorias anuales que el Consejo Regulador de la indicación geográfica protegida Brandy de Jerez emitió desde su nacimiento, en 1989, hasta el pasado 2008. En este contexto, especial atención merece destacar la labor de recopilación, ordenación y síntesis que el presente estudio realiza de la gran red de normas legales que existen a nivel internacional, comunitario y nacional en torno a las indicaciones geográficas.

Para Pérez Serrano (2004:81) el estudio de caso consiste en probar de modo profundo y analizar con intensidad el fenómeno diverso que constituye el ciclo vital de la unidad. Definición esta que encaja con nuestra intencionalidad de someter a contrastación empírica el comportamiento relacional de un caso concreto, el del Consejo Regulador del Brandy de Jerez ${ }^{1}$, a través de las memorias oficiales como fuentes estadísticas oficiales.

El caso estudiado también se limita a su ciclo vital, ya que se analizan sus primeros 20 años de historia. Este aspecto resulta especialmente relevante ya que el estudio de casos se manifiesta como uno de los métodos idóneos para el estudio de una entidad u organización en un periodo de tiempo determinado. Además, este análisis del comportamiento relacional del CRDEBJ hace necesario abordar el estudio de la organización en su contexto real.

Este aspecto justifica la utilización del estudio de casos ya que según autores como Yin (1988) o Pérez Serrano (2004:80-83) el método del estudio de casos es una investigación empírica que estudia un fenómeno actual dentro de su contexto real, dirigido a situaciones en las que los límites entre el fenómeno y el contexto son confusos, y usa múltiples fuentes de evidencia.

Se aplica en primer lugar a nuestra investigación el método del estudio de casos, centrado en el diseño de caso único. Esta metodología permite dar una validez lógica al problema principal planteado en la presente investigación: abordar el estudio de las indicaciones geográficas desde la perspectiva de la gestión de las relaciones públicas en el caso concreto de la DEBJ como estrategia de gestión de las relaciones del Consejo Regulador que la tutela, el CRDEBJ.

Desde esta perspectiva solo el estudio de caso, permite el conocimiento en profundidad del funcionamiento de una organización desde la perspectiva de la gestión de sus relaciones con los públicos de su entorno desde una perspectiva diacrónica.

Para llevar a cabo el estudio de caso, que se complementa con el método de análisis de contenido, nos hemos basado en las técnicas de la observación, la entrevista y la utilización de fuentes de datos secundarios.

1 En adelante, CRDEBJ 


\subsection{Análisis de contenido}

Para Bardin (1986:7) el análisis de contenido es un conjunto de instrumentos metodológicos aplicados a discursos (contenidos y continentes) extremadamente diversificados. Es un conjunto de técnicas de análisis de comunicaciones (Bardin, 1986:23) destinada a formular, a partir de ciertos datos, inferencias reproducibles y válidas que puedan aplicarse a su contexto (Krippendorf, 1990:28). Un texto puede ser analizado de forma cualitativa, tratando su contenido desde su globalidad y de forma cuantitativa, subdividiéndolo en elementos homogéneos que, una vez tratados fundamentalmente a través de técnicas estadísticas, van a ser relacionados entre sí. La utilización del criterio cualitativo da lugar al análisis textual de tipo cualitativo. La utilización del criterio cuantitativo da lugar al análisis de contenido, que aplica procedimientos de descomposición de los textos para codificarlos en una matriz de datos que posteriormente es analizada a través de técnicas estadísticas (Corbetta, 2007:390).

De este modo, como método exhaustivo de conocimiento (Krippendorf, 1990), permite completar la presente investigación a través de la extracción y cuantificación, para su posterior análisis, de aquella información clave contenida en las memorias de los primeros 20 años de historia del CRDEBJ. En este sentido, el análisis de contenido aporta a nuestra investigación la posibilidad de construir cómo, y a través de qué técnicas, se conforma el comportamiento comunicacional del CRDEBJ a lo largo del período analizado desde una perspectiva diacrónica. Para que el análisis de contenido pueda ser aplicado de forma eficaz al citado cuerpo de estudio se han diseñado diversas herramientas cuyo objetivo es descomponer el texto para codificarlo en una matriz de datos que permita su posterior análisis. Así, atendiendo a las referencias de los autores consultados (Bardin, 1986; Kripendorff, 1990; Mayntz, Holm y Hübner, 1993; Ander-Egg, 1995; Cea, 2001 y Corbetta, 2007), la ordenación, la clasificación, el análisis de la estructura, la lectura sistemática atendiendo a los índices A, B y C elaborados, y la tabulación en una matriz de datos, son las principales herramientas diseñadas para ejecutar el análisis de contenido al citado cuerpo de estudio.

\subsection{Recogida de datos}

\subsubsection{La observación}

Para González (1997) la observación es uno de los procedimientos que permiten la recolección de información que consiste en contemplar sistemática y detenidamente cómo se desarrolla la vida social. Alude al conjunto de métodos establecidos para la observación directa de sucesos que ocurren de un modo natural. Esta definición implica dos consideraciones principales: en primer lugar que los datos se recogen cuando ocurre el suceso, sin que ello implique la imposibilidad de que sea grabado o recogido para su posterior análisis; en segundo lugar, significa que el suceso no es creado, mantenido o finalizado exclusiva- 
mente para la investigación. Según establece la citada autora, es la técnica de investigación más importante empleada en la sociología de forma que ninguna otra técnica puede reemplazar el contacto directo del investigador con el campo de estudio y afirma (González, 1997:124):

\footnotetext{
«Ninguna otra técnica es capaz de sugerir tantas ideas nuevas. Es difícil imaginar un estudio serio en el que la observación no desempeñe ningún papel. Cualquier investigación debería empezar por una fase previa de observación».
}

En este sentido, en el ámbito concreto de la presente investigación, la técnica de la observación es utilizada tanto en la fase previa a la selección y formulación del problema a investigar, como en la fase de recolección de datos que nos ocupa en este epígrafe. Existen dos tipos de observación: la observación sistemática y la participante (González, 1997:126). Para García (1981) la observación sistemática hace referencia a la observación y registro de comportamientos y acciones previamente especificados. Se puede aplicar siempre que el ámbito seleccionado se produzca en situaciones públicas y que permita que el investigador realice la observación sin participar directamente en la situación. Sin embargo, a diferencia de la observación sistemática, en la observación participante el investigador forma parte de los sucesos que se observan. En este sentido, en el ámbito concreto de la presente investigación la técnica concreta utilizada es la de la observación sistemática, ya que en palabras de González (1997:126) «se recomienda la utilización de los métodos de observación cuando se formulan hipótesis nuevas y cuando se realiza investigación exploratoria y descriptiva». Como apuntamos anteriormente, la naturaleza de nuestro diseño de investigación, que combina el estudio exploratorio con el estudio descriptivo - explicativo, razón que justifica utilización de la técnica de la observación sistemática en el presente trabajo de investigación. La observación sistemática supone que los sucesos que se observan son seleccionados, anotados y codificados.

En relación a estas últimas consideraciones reseñamos a continuación tanto la selección de la muestra sobre la que se aplica la técnica así como el diseño de la herramienta a través del establecimiento de los sucesos que previamente habían sido seleccionados para ser observados en la recogida de datos.

\subsubsection{Diseño de la herramienta y aplicación}

Dado que la técnica de la observación es aquí empleada para poder aplicar el método del estudio de caso en el diseño de caso único: el CRDEBJ, la muestra observada es el propio CRDEBJ. Así, los ítems seleccionados que se exponen a continuación y que forman parte del diseño de la herramienta para que guíen y orienten la observación sistemática como técnica de investigación, se aplican sobre todos los componentes humanos y físicos, mobiliarios e inmobiliarios, que conforman el CRDEBJ. 
En la etapa previa a la formulación del tema de investigación la observación se utiliza para determinar tanto el nivel de calidad, la cantidad, la asequibilidad y el orden que la documentación que los archivos, así como el grado de acceso al campo de estudio a través del contacto con los componentes de ambos Consejos Reguladores sitos en Jerez de la Frontera, para determinar sobre qué institución centrar la investigación a través, en primer lugar, del estudio de caso. En esta etapa, se establecen una serie de ítems observables que permitieran guiar la recolección de los datos hacia los intereses marcados por los objetivos de la investigación. En este sentido, después de una primera toma de contacto con el CRDEBJ, procedemos a diseñar una relación de ítems observables que se organizan en dos grandes bloques: organización y archivos, por una parte, y material de comunicación, por otra.

En primer lugar, en relación a la organización interesan tanto su estructura jerárquica y composición como su aspecto externo como edificio sede de la organización. En este sentido interesan:

\section{Composición y estructura jerárquica del CRDEBJ:}

Número de componentes de la organización.

Ambiente en el que se desarrolla el trato entre los miembros de la institución.

Interés de los miembros de la organización por facilitar la información requerida por la investigadora para el desarrollo y ejercicio del trabajo de investigación.

Grado de acogimiento e interés por parte de los representantes de la institución porque un trabajo de investigación conducente al título de doctor se realice sobre su organización.

Grado de conocimiento de cada uno de los componentes de la organización sobre la institución.

Grado de asunción de la misión y objetivos de la organización.

Grado de asequibilidad por parte de la investigadora a cada uno de los miembros de la organización en relación al cargo ocupado en relación a la estructura jerárquica establecida en el organigrama.

Estructura jerárquica del CRDEBJ.

\begin{tabular}{|l|}
\hline \multicolumn{1}{|c|}{ Aspecto general exterior de la sede de la institución } \\
\hline Ubicación física y emplazamiento. \\
\hline Se comparte o no el edificio con otras organizaciones. \\
\hline Aspecto general interior: disposición de mobiliario y decoración. \\
\hline Ubicación del archivo del CRDEBJ en relación al resto de las instalaciones de la organización. \\
\hline
\end{tabular}


En segundo lugar, en relación a los archivos y el material de comunicación de la organización se señalan los siguientes ítems observables:

\begin{tabular}{|l|}
\hline \multicolumn{1}{|c|}{ Material de comunicación } \\
\hline Aspecto general del archivo de la institución. \\
\hline Grado de cuidado y archivo de la documentación \\
\hline Clasificación y orden o no de los documentos. \\
\hline Grado de asequibilidad a los documentos requeridos para la investigación. \\
\hline Documentación extraviada, deteriorada o no disponible. \\
\hline $\begin{array}{l}\text { Recopilación, o no, y archivo, en su caso, de documentos relativos a las informaciones vertidas } \\
\text { sobre la organización o el sector en los medios de comunicación. }\end{array}$ \\
\hline $\begin{array}{l}\text { Observación, manipulación, visionado y lectura de todo el material de comunicación disponible } \\
\text { para la aplicación de los Ítems M1 y M2, correspondientes al análisis de contenido y que se descri- } \\
\text { birán con posterioridad en el epígrafe dedicado a tal efecto. }\end{array}$ \\
\hline
\end{tabular}

Esta técnica fue llevada a desde enero hasta marzo de 2009.

\subsubsection{La entrevista}

Como procedimiento científico para la recolección de datos, la entrevista hace referencia al proceso de interacción donde la información fluye de forma asimétrica entre dos roles bien diferenciados, de los que uno pregunta y el otro responde (González Río, 1997:154). En nuestra investigación, la entrevista como técnica complementaria, permite tener acceso a información específica y concreta que no se encuentra contenida en las fuentes de datos secundarios que suponen el cuerpo principal de análisis. No obstante existen diferentes tipos de entrevista. Uno u otro tipo deberán ser seleccionados en virtud del que sea más apropiado atendiendo al propósito de la investigación.

Corbetta (2007:34) establece dos tipos de instrumentos para obtener información planteando preguntas a los sujetos: la entrevista cuantitativa y la entrevista cualitativa. La selección de una u otra técnica debe hacerse en función de los problemas que plantea y la finalidad de la investigación que se realiza. A este respecto afirma González (1997:156):

«La existencia de diferentes tipos de entrevista permite una mayor capacidad de elección técnica, pero no la arbitrariedad. Existe una correspondencia directa entre el tipo de entrevista a emplear y la finalidad de la información que se va obtener en el desarrollo de la investigación».

Frente a otro tipo, la entrevista cualitativa tiene la particularidad de permitir «obtener datos preguntando a un entrevistado conociendo la individualidad de la persona entrevistada y de ver el mundo con sus ojos» (Corbetta, 2007:344) 
lo que implica que el sujeto entrevistado es seleccionado por las posibilidades de información que, en profundidad, que puede aportar sobre el tema de la investigación.

En este sentido, para Corbetta (2007), la entrevista cualitativa reúne las siguientes condiciones:

- es una conversación solicitada de manera explícita por el entrevistador

- que se realiza normalmente a través de una cita programada

- que se realiza a una serie de sujetos seleccionados

- según un plan sistemático de recogida de datos.

Es decir, el entrevistado no es ocasional sino elegido por unas características determinadas: vivencias, experiencias, etc.; no son entrevistas esporádicas, sino realizadas a un número de sujetos tal que permita inferir informaciones generalizables a una población más amplia (aunque no se pretende llegar a muestras representativas en un verdadero sentido estadístico); debe ser una conversación guiada, en la que el entrevistador establece el tema y controla que su desarrollo responda a los fines cognitivos que se ha marcado.

En este sentido, para completar la recolección de datos que permita el mejor desarrollo de nuestra investigación se ha optado por la entrevista cualitativa. No obstante para poder aplicarla con garantía, hemos tenido en cuenta las tres características fundamentales de la entrevista cualitativa como técnica de recolección de datos. Para Corbetta (2007:345-349), estas características son:

- Falta de estandarización

- Predominio del contexto de descubrimiento frente al de justificación.

- Falta de muestra representativa.

- Criterio centrado en el sujeto frente a criterio centrado en las variables.

Estas características hacen de la entrevista cualitativa el mejor instrumento, junto a la observación y a las fuentes de datos secundarios, para complementar el estudio de caso que se plantea como método general de la presente investigación. Además, como afirma González (1997: 157) con carácter general:

\footnotetext{
«Las entrevistas en las que la forma de recoger los datos no está estructurada y la dirección no se halla estandarizada son útiles en las investigaciones exploratorias, donde se tiene poco conocimiento del fenómeno en estudio. (...) La dimensión principal de una entrevista no estandarizada y no estructurada es la recolección de la información».
}

A su vez, aunque la entrevista cualitativa se caracteriza por ser un instrumento flexible y abierto (Corbetta, 2007:350), la flexibilidad puede presentar varios grados. En este sentido, la entrevista puede ser estructurada, semiestructurada y no estructurada. 
Atendiendo a las necesidades de información que requiere nuestra investigación, hemos optado por la entrevista semiestructurada basada en un guión abierto que recoge los temas que se deben tratar a lo largo de la entrevista. Para Corbetta (2007:353) la entrevista semiestructurada puede ser una lista de temas a tratar, consideración esta que tiene en cuenta la entrevistadora a la hora de diseñar la herramienta. Como técnica de recogida de datos, es una herramienta a través de la que se puede obtener la información que subyace en los discursos estructurados, resaltando las opiniones, las dudas y las contradicciones, algo difícil de captar a través de un cuestionario de respuesta cerrada. La información obtenida de esta manera es mucho más rica en matices y permite una aproximación más completa al fenómeno estudiado.

\subsubsection{Diseño de la herramienta y aplicación}

La técnica de la entrevista ha sido aplicada a dos sectores claramente diferenciados en función del ámbito directivo y ejecutivo en la estructura jerárquica del CRDEBJ. En el ámbito directivo, como se adelantaba anteriormente, se ha realizado una entrevista en profundidad al Presidente del CRDEBJ desde su fundación hasta la fecha de la realización de la entrevista. En el ámbito ejecutivo, se mantuvo una entrevista con la persona responsable a nivel ejecutivo de las tareas de comunicación llevadas a cabo por el CRDEBJ desde su fundación hasta 2009. En ambos casos nos encontramos ante lo que Corbetta (2007:358) denomina observadores privilegiados. La entrevista a observadores privilegiados es aquella en la «que el investigador entrevista a sujetos no como parte del fenómeno a estudiar, sino como conocedores expertos del fenómeno, con una visión directa y profunda del mismo que los sitúa en una posición de observación privilegiada».

Dada la naturaleza del problema investigado, y que estas técnicas de la entrevista y la observación se hacen como auxiliares al análisis de los datos de fuentes secundarias, la entrevista a observadores privilegiados es la que mejor se ajusta a las necesidades de la investigadora en función de la obtención de datos para la consecución de los objetivos fijados para el análisis empírico que no pueden ser recogidos a través de las fuentes de datos secundarios. En este sentido, estas dos personas, desde nuestra perspectiva se manifiestan como los elementos clave que permiten acometer el estudio de caso del CRDEBJ desde la técnica de la entrevista.

A cada uno de los observadores privilegiados grupos se aplica una entrevista en profundidad semiestructurada diferente. En este sentido se diseñan entrevistas diferentes cuyo diseño gira en torno a las preguntas que se exponen a continuación. 


\begin{tabular}{|c|c|}
\hline \multicolumn{2}{|r|}{ Entrevista A: nivel DIRECTIVO. } \\
\hline Primera cuestión: & $\begin{array}{l}\text { El CRDEBJ como organización (naturaleza jurídica, grado de } \\
\text { dependencia en su caso con la administración del estado, etc.). }\end{array}$ \\
\hline Segunda cuestión: & $\begin{array}{l}\text { Contexto, razones e intereses que mueven a la adopción de una DE al } \\
\text { sector productivo del Brandy de Jerez. }\end{array}$ \\
\hline Tercera cuestión: & $\begin{array}{l}\text { Aportaciones fundamentales derivadas de la adopción de la DE (en } \\
\text { relación al producto Brandy de Jerez, a las bodegas que lo producen, los } \\
\text { públicos, etc.). }\end{array}$ \\
\hline Cuarta cuestión: & $\begin{array}{l}\text { Las relaciones públicas como la disciplina que ayuda a gestionar de forma } \\
\text { estratégica las relaciones con los públicos de su entorno, cree que la } \\
\text { adopción de una DE y, consecuentemente del CRDEBJ, ayuda al Brandy } \\
\text { de Jerez a } \\
\text { posicionarse de forma positiva en la sociedad de su entorno: } \\
\text { a. Posible consideración de la DE como estrategia que permite } \\
\text { una gestión adecuada de las relaciones entre el CRDEBJ } \\
\text { que la tutela y su entorno. } \\
\text { b. Posibles consideraciones entre la protección jurídica que pro- } \\
\text { porciona la adopción de la DE y la reputación del producto } \\
\text { al que ampara. } \\
\text { c. Permite la DE, a través del CRDEBJ, lanzar mensajes de legi- } \\
\text { timación del producto al margen de marcas. }\end{array}$ \\
\hline Quinta cuestión: & $\begin{array}{l}\text { Mensaje fundamental a trasladar a través de la comunicación emitida por } \\
\text { el CRDEBJ. }\end{array}$ \\
\hline
\end{tabular}

\section{Entrevista B: nivel EJECUTIVO.}

\begin{tabular}{|l|l|}
\hline Primera cuestión: & $\begin{array}{l}\text { Públicos y mensajes organizacionales clave que impregnan la comunicación } \\
\text { del CRDEBJ. }\end{array}$ \\
\hline Segunda cuestión: & $\begin{array}{l}\text { Otras relaciones con los medios de comunicación no expresados en las } \\
\text { memorias. }\end{array}$ \\
\hline Tercera cuestión: & Estado del archivo del CRDEBJ. \\
\hline Cuarta cuestión: & $\begin{array}{l}\text { Otras acciones de comunicación no reveladas en las memorias, no se } \\
\text { refieren otras acciones al margende las ya mencionadas. }\end{array}$ \\
\hline Quinta cuestión: & $\begin{array}{l}\text { Otro material relacionado con la gestión de la comunicación como } \\
\text { material promocional al que se hace referencia en las memorias. }\end{array}$ \\
\hline
\end{tabular}

Las entrevistas se han llevado a cabo en junio y agosto de 2010. 


\subsubsection{La utilización de fuentes de datos secundarios}

En el proceso de investigación se distinguen dos tipos fundamentales de datos: los primarios y los secundarios (González, 1997:244). Los primarios hacen referencia a informaciones recogidas de primera mano, mientras que los secundarios son datos extraídos de los datos originales recogidos por otras personas. Los datos secundarios aluden a informaciones que no han sido producidas de forma explícita para los objetivos de la investigación. Como se decía en páginas anteriores, las fuentes de datos secundarios suponen el cuerpo de estudio fundamental de la presente investigación a través principalmente de las memorias oficiales anuales del CRDEBJ, la legislación y, de forma complementaria, la revista Brandy de Jerez al día.

Resulta de especial interés para la investigadora el siguiente fragmento de González (1997:244) en relación a la validez de los datos secundarios, fundamentalmente en cuanto a las preguntas planteadas para la investigación y la capacidad de dicha información de resolver las cuestiones planteadas que tratan de resolverse a través del proceso de la investigación. En este sentido afirma González (1997:244):

«El principio básico para el uso de datos secundarios consiste en ser flexible a la hora de plantearse las preguntas en una investigación. Lo ideal es que una idea o una hipótesis pueda ser planteada de forma que el material disponible ya recopilado contenga la respuesta a esa pregunta».

Además, según la autora, la utilización de este tipo de fuentes proporciona tres ventajas claras a la investigación: economía, permite el análisis en series temporales y no requiere la cooperación de terceros.

Es especialmente relevante para nuestra investigación la segunda de las ventajas ya que los datos secundarios, al ser normalmente un tipo de información recogida periódicamente, además de hacer posible, como se decía en el párrafo anterior, el análisis en series temporales, permite analizar el suceso investigado a través del tiempo. Las fuentes secundarias ofrecen información derivada del uso de documentos como técnica para la recogida de datos. Para Corbetta (2007:375-400) los documentos pueden ser personales o institucionales. Los documentos personales son autobiografías, diarios, cartas y testimonios orales. Los documentos institucionales se subdividen en medios de comunicación; narrativa, textos pedagógicos y cuentos populares; material judicial; documentos de la política; documentos empresariales y administrativos y huellas físicas. Para González (1997:245), las fuentes secundarias se pueden clasificar en cinco categorías:

- Documentos públicos o archivos oficiales.

- Documentos personales.

- Información de los medios de comunicación de masas.

- Medios audiovisuales.

- Materiales no verbales.

- Archivo de datos sociológicos. 
3.2.3.1. Descripción del material obtenido a partir de las fuentes de datos secundarios y ámbito de recogida:

Las fuentes de datos secundarios utilizadas en la presente investigación son:

- Memorias anuales de actividades del CRDEBJ

- Revista Brandy de Jerez al día

- Fuentes bibliográficas.

- Normas legales internacionales, comunitarias y nacionales fundamentales en relación a las indicaciones geográficas.

3.2.3.2. Diseño de las herramientas para la recogida de datos a través de las memorias de actividades emitidas por el CRDEBJ entre 1989 y 2008.

Para extraer de las memorias de actividades, emitidas por el CRDEBJ correspondientes al periodo investigado, se diseña una herramienta de análisis en cinco fases:

- Primera lectura de la memoria completa, que permite obtener una visión global tanto del valor documental como de la composición de la memoria.

- Segunda lectura comprensiva en relación a los objetivos generales y específicos de la investigación, que permite identificar los tres aspectos clave de las memorias en relación a los objetivos de la investigación: estructura, introducción y área de comunicación.

- Análisis de la estructura de las memorias analizadas, que tiene como objetivo analizar la estructura de las memorias en relación, fundamentalmente al espacio que se le dedica al área de la comunicación en relación a la memoria completa.

- Análisis cualitativo de las introducciones de las memorias objeto de estudio.

- Análisis del área dedicada a las actividades de comunicación llevadas a cabo por el CRDEBJ.

3.2.3.3. Diseño de las herramientas para la recogida de datos a través de las normas legales

Para la recopilación de datos relevantes para la investigación, las normas legales son reunidas en tres grupos:

- Normas legales internacionales y comunitarias

- Normas legales nacionales

- Normas legales específicas reguladoras de la DEBJ. 
En primer lugar para la recogida de datos procedentes de las normas legales internacionales y comunitarias se confeccionan dos campos semánticos: campo semántico 1, en torno al concepto de reputación; y campo semántico 2, en torno al concepto de confianza. La extracción de datos a través del diseño de estos campos semánticos se hace a través de la identificación en los textos sobre los que se aplica de los términos reputación, notoriedad, imagen y prestigio en el caso del campo semántico 1 y de los términos credibilidad, confianza, calidad y protección en el caso del campo semántico 2.

3.2.3.3. Diseño de las herramientas para la recogida de datos a través de las fuentes bibliográficas y la revista Brandy de Jerez

Tanto las fuentes bibliográficas como la revista Brandy de Jerez, serán utilizada de forma auxiliar y complementaria al resto de herramientas diseñadas para la extracción de datos. Para ello se seleccionará aquella información que, de forma directa o indirecta, puedan ser objeto de interés para el desarrollo del estudio empírico que se lleva a cabo en esta investigación.

\subsubsection{Diseño de las herramientas para aplicar el análisis de contenido a} las áreas de comunicación de las memorias de actividades del CRDEBJ.

El análisis de contenido, llevado a cabo en los meses de julio y agosto de 2010, se aplica sobre los epígrafes, correspondientes a las actividades del área de comunicación, reseñadas en cada una de las veinte memorias anuales emitidas por el CRDEBJ (memorias correspondientes al periodo temporal investigado entre los años 1989 y 2008, ambos incluidos).

El objetivo de la aplicación del método del análisis de contenido a través de las técnicas que se reseñan a continuación, es extraer las acciones de comunicación realizadas por el CRDEBJ en el período temporal investigado, atendiendo, entre otros factores que serán descritos posteriormente, a la frecuencia de su realización.

Dichos datos suponen la información clave que permite construir el comportamiento comunicacional llevado a cabo por el CRDEBJ. El procedimiento desarrollado para el análisis de contenido se compone de cinco fases:

- Clasificación y preparación del material por lustros.

- Lectura sistemática de las áreas de comunicación contenidas en cada una de las veinte memorias de actividades del CRDEBJ del período analizado.

- Tabulación informática de las acciones en una matriz de datos original por lustros, atendiendo a los factores de frecuencia F1 y F+. 
- Aplicación de los Índices A, B y C sobre la matriz de datos original por lustros, según los criterios establecidos.

- Tabulación informática en una matriz de datos definitiva, resultante del proceso descrito.

Además de servir a los objetivos de la investigación previstos ya descritos, tanto la observación, como la entrevista y la utilización de las revistas Brandy de Jerez al día, sirven como fuentes auxiliares que permiten completar la aplicación de los índices A, B y C para la elaboración de la matriz de datos definitiva.

\section{El label de calidad}

4.1. ¿Qué supone un label de calidad? Éxito de los estándares de gestión de calidad.

Comenzamos exponiendo que por label de calidad se entienden los siguientes tipos:

- Denominación de Origen Protegida (DOP): Supone el nombre de una región o zona utilizada para denominar un producto agrícola o alimentario que se produce en esa área y que presenta una calidad y/o características que se derivan del medio geográfico en el que se obtiene.

- Indicación Geográfica Protegida (IGP): Se trata de una región o zona que se emplea para designar un producto agrícola o alimentario que posee una reputación reconocida y que puede relacionarse con el lugar de origen en el que se ha llevado a cabo, al menos, la producción, transformación o elaboración.

- Especialidad Tradicional Garantizada (ETG): Hace referencia a productos agrícolas o alimentarios que poseen unas características singulares que permiten su distinción con respecto a otros productos similares, bien debido a la utilización de materias primas tradicionales o a sus presentaciones tradicionales o a la utilización de métodos de producción y/o transformación tradicionales.

A nivel teórico, y desde una perspectiva de análisis organizativa, no existe un claro consenso entre los expertos a la hora de establecer cuáles son las razones explicativas principales de la gran difusión experimentada por las normas y la heterogeneidad existente en los países industrializados en cuanto a su grado de penetración.

En la amplia literatura organizativa existente, tanto en los estudios teóricos como en los empíricos basados en la opinión de los directivos se subrayan las bondades de la aplicación de estos estándares para la eficiencia empresarial, aunque también existen planteamientos y estudios críticos, que subrayan las supuestas debilidades de la implantación de estos estándares internacionales, 
tildándolas de ser una moda, un factor de rigidez, una fuente de descalificación para el trabajador, un retorno al taylorismo, de tener un costo elevado y, fundamentalmente, de establecer unos modelos de gestión que aumentan el papeleo y la burocracia. Existen también estudios cuantitativos fundamentados en datos agregados, en este caso basados en la utilización de bases de datos comerciales de información económico-financiera empresarial. Así, Neumayer y Perkins (2005) subrayan que existen dos grupos de motivaciones fundamentales que llevan a las empresas a implantar estas normas, a certificarlas y protegerlas: por un lado, los motivos internos relacionados con la eficiencia y, por otro lado, los motivos externos o institucionales, relacionados con la presión social que ejercen diversos actores para que se adopten estas prácticas de gestión de empresas.

Así, también desde una perspectiva académica la apuesta por la calidad aparece como una de las oportunidades más sólidas con las que cuenta los sectores agroalimentarios español y europeo para incrementar y fortalecer su presencia en los mercados internos y exteriores. En un contexto caracterizado por competencia (no siempre leal), dificultades en el comercio exterior de productos alimentarios y las sucesivas crisis (de carácter sanitario) que pueden dañar la confianza de los consumidores, el segmento de los alimentos de calidad amparados por algún tipo de figura de protección es el que presenta unos índices más sostenidos de crecimiento. De hecho, esa apuesta por la calidad puede ser vista como una orientación estratégica de las políticas alimentarias, promoviendo modelos de agricultura donde los tradicionales parámetros de cantidad vayan dando paso paulatinamente a valoraciones esencialmente cualitativas.

Un label de calidad (sea IGP, DOP o ETG) supone, de algún modo no producir siempre mayores cantidades de producto, sino de promocionar una oferta de alimentos de alta calidad que, además, permitan una adecuada gestión de los recursos sentando las bases de la gestión estratégica de la empresa, su misión y visión.

\subsection{Antecedentes inmediatos de las indicaciones geográficas como fórmulas de protección.}

La evolución histórica hace que la designación por el origen siga desarrollándose vinculada, además, a la notoriedad o reputación de determinados productos. $\mathrm{Y}$ es que los antecedentes de las denominaciones geográficas como marcas de calidad son relativamente recientes... Si nos centramos específicamente en la indicación geográfica, la utilización del nombre del lugar de procedencia para vender y ubicar un producto y así distinguirlo de otros de similar especie, bien como mera información, bien por motivos arancelarios, bien para el aporte de prestigio es tan antigua como el origen de la civilización (Couillerot, 2000 en Granados, 2004:5). De hecho, en la propia Biblia, el libro más antiguo conocido, se muestra «la existencia de esta práctica, con alusiones a la madera de cedro del Líbano, o a los vinos de Engaddi, de Jelbon y del Líbano entre otros (...). 
Especialmente significativo resulta el estudio de dos de las civilizaciones griega y romana cuando, según Maroño (2002: 21):

«Evidencian el aprecio de éstos por el vino de Falerno, si bien también gustaban del vino de Sorrente, de Cos, etc., e igualmente, conocían el jamón de Iberia, y el de la Galia, los papiros del Valle del Nilo o los dátiles de Egipto»

En este sentido, destacamos también que, en línea con las afirmaciones de Schiavone (2003:17), ya en el siglo XIV, el queso Roquefort adquirió una notoriedad que conserva hasta la actualidad, bajo el nombre de su zona de origen, siendo objeto incluso de una cédula real de Carlos V concedida a los habitantes de Roquefort. Posteriormente, el desarrollo cada vez más intenso del comercio, derivado de la Revolución Industrial, provoca el incremento de la demanda de productos reputados. Este contexto, se convierte en la base para un conflicto de intereses entre aquellos que querían conservar un derecho adquirido gracias a las condiciones climáticas, tipo de suelos, formas de cultivar los productos o fabricarlos y aquellos que trataban de utilizar denominaciones usurpadas a fin de sacar un provecho ilegítimo (Schiavone, 2003:17).

\subsubsection{El nacimiento de la protección en el ámbito internacional del sector vitivinícola}

En torno al 1300, comienzan a surgir en Francia algunas normas que establecían que no se daría a los vinos de una región otro nombre que aquel donde sea creado (Schiavone, 2003:17).

No obstante, a pesar de estas referencias, desde una perspectiva histórica, las primeras diferenciaciones de calidad como tal, ligadas al origen geográfico de los alimentos, no aparecen hasta finales del siglo XIX (Barco, 2007): es a finales del siglo XIX cuando se inicia una somera protección de estas denominaciones como consecuencia de las ya existentes denominaciones de origen. Es entonces cuando emerge la búsqueda de una tímida protección legal de determinados productos originales y característicos de ciertas zonas que sufrían procesos de imitación que desembocaban en competencia fraudulenta en los mercados (Martín Cerdeño, 2006). En concreto, este fenómeno aparece en torno a la producción de vino y aceite en el sur de Europa debido a la especial sensibilidad de la vid y el olivo a las condiciones medioambientales del entorno geográfico donde se cultivan. Países como Portugal, Francia, Italia o España son los pioneros en demostrar una mayor preocupación por la calidad diferenciada de los productos agroalimentarios asociada a una procedencia geográfica determinada (Martín, 2006).

Para autores como Schiavone (2003) este incipiente desarrollo normativo comienza en 1870 cuando los viñedos franceses son atacados por una plaga de filoxera (un insecto que ataca las vides) que provocó un desabastecimiento importante de caldos. 
En este contexto, y debido a los condicionamientos climatológicos, los viñedos del sur de Francia se recuperaron más rápido de lo esperado, lo que provocó que muchos vinos abusaran de nombres reputados y célebres como Burdeos y Borgoña siendo, en realidad, procedentes de otras áreas geográficas (Schiavone, 2003). En concreto, el nacimiento de las fórmulas de protección internacionales nace con el Convenio de la Unión de París en 1978 y evoluciona con el denominado El Arreglo de Madrid para la represión de las indicaciones falsas o falaces de procedencia de las mercancías de 1891. Tras la Exposición Universal de París se presentó un proyecto jurídico para constituir una organización, denominada Unión General para la Protección de la Propiedad Industrial que, a través de la celebración de un congreso anual, resolviese una serie de problemas que se planteaban en relación al comercio internacional. Fue en 1883 cuando se aprobó el convenio de la Unión de París para la Propiedad Industrial en el que se incorpora un listado de sanciones para aquellos productos que indicasen una procedencia, localidad o región siendo falso.

Este conflicto de intereses se resolverá con el nacimiento de las primeras normas reguladoras para la protección de determinados productos reputados por su origen geográfico o los métodos empleados en su elaboración que tendrán su eclosión ya en el siglo XX (López Benítez, 1996:23) como se explicita a continuación.

Si nos centramos en el caso español, en la legislación española, la Ley, de 16 de mayo de 1902, sobre Propiedad Industrial y Comercial ya aportaba el libre uso (verdadero y sin llevar a falsedades) de marcas colectivas con el nombre geográfico del lugar de fabricación, elaboración o extracción de un producto. Sin embargo, es en 1932 cuando se produce realmente la introducción de la denominación de origen en el ordenamiento jurídico español. Pero, ciertamente, el fenómeno de las indicaciones geográficas protegidas no surge como tal y con pleno desarrollo en torno al sector vitivinícola hasta 1933 con el denominado Estatuto Republicano del Vino, desarrollándose posteriormente la Ley del vino de 1970, que se desarrolla en el Estatuto de lo vino de 1972. En la actualidad, el sector vitivinícola se regula de forma general por la Ley 24/2003.

Estas normativas se complementarán posteriormente en el ámbito internacional con El Arreglo de Lisboa relativo a la protección de las denominaciones de origen y a su registro internacional de 1958 y el Los Aspectos de los Derechos de la Propiedad Intelectual relacionados con el comercio perteneciente al Acuerdo de la Organización Mundial del Comercio de 1994.

Estas cuatro normativas suponen el marco jurídico internacional fundamental sobre el que se sustenta el posterior desarrollo de las fórmulas de protección que se desarrollarán en cada uno de los países, tal y como sucede en el caso de España, así como en la comunidad europea. 


\section{Resultados. Las indicaciones geográficas desde el punto de vista de las RR.PP.}

Si partimos del concepto de relaciones públicas, podemos afirmar que puede definirse como «aquella disciplina científica que se encarga de la dirección y gestión de la comunicación de una entidad u organización física o jurídica con los públicos de su entorno» (Grunig y Hunt, 2000:52). Y es que, atendiendo a las afirmaciones de estos autores, el mayor peso de la definición recae en la dirección y gestión de la comunicación al afirmar que las relaciones públicas son una función directiva de gestión de la comunicación entre una organización y sus públicos y no cualquier otro departamento (sea o no residual).

Bernays (1998) y Seitel (2002) aportan a esta definición el doble sentido o bidireccionalidad de las RR.PP., dado que público interpreta a la organización y, al mismo tiempo, organización interpreta a sus públicos. Así, en palabras de Seitel (2002:14) «los profesionales de las relaciones públicas son, fundamentalmente, intérpretes. Por un lado, deben interpretar las filosofías, políticas, programas y prácticas de su dirección dirigidos al público; por otro, deben trasladar las actitudes del público a su dirección». Destacan especialmente la definición de Long y Hazelton que, poniendo de manifiesto la coincidencia con Grunig y Hunt (2000) en poner el punto de atención de la definición en la función directiva de comunicación de la actividad, describen las relaciones públicas como «una función directiva de comunicación a través de la cual las organizaciones se adaptan a, alteran o mantienen su entorno con el objetivo de lograr sus fines como organización» (Wilcox, Autt, Agee y Cameron (2000:4).

Pero, ¿por qué una definición de RR.PP.? Porque desde esta investigación defendemos que desde una perspectiva comunicacional, son las relaciones públicas las encargadas del estudio, gestión e implantación de estos sistemas de comunicación organizacionales bidireccionales que tratan de generar un clima de entendimiento mutuo que permita a la organización (empresa privada o institución pública) crecer y desarrollar su actividad en óptimas condiciones en un contexto socioeconómico, político y cultural compuesto por sus diferentes sectores de públicos.

Estos sistemas de comunicación organizacionales se caracterizan esencialmente por su naturaleza y finalidad. Así, en cuanto a su naturaleza, las relaciones públicas se caracterizan por establecer y desarrollar un proceso de comunicación organización-públicos-organización bidireccional, basado en el establecimiento de un diálogo de utilidad entre la organización y su entorno. Y en relación a su finalidad, las relaciones públicas se caracterizan por tratar de generar un clima de entendimiento mutuo por el cual la organización pueda llevar a cabo sus actividades cotidianas con los mejores resultados posibles. De esta forma, las relaciones públicas permiten engrasar los engranajes de la organización en pos de optimizar sus resultados organizacionales, su misión y objetivos en la sociedad en la que está inserta. 
Los resultados del análisis de los datos recogidos a través de la observación se organizan en dos bloques:

Resultados derivados de los ítems observables sobre la organización:

- Destaca una composición y estructura jerárquica del CRDEBJ sencilla en relación al número de componentes de la organización, así como un trato muy cuidado entre ellos.

- En relación al aspecto del edificio destaca un aspecto exterior propio de nuevo de los organismos públicos, siendo bueno y cuidado

En relación a los archivos y el material de comunicación de la organización se señalan lo siguiente:

- Se tiene total acceso a la información.

- No se conservan determinados ejemplares de la revista Brandy de Jerez al día

- No existe prácticamente ninguno de los materiales enviados a los medios de comunicación: notas de prensa, etc.

En relación a los resultados de las entrevistas a nivel directivo y ejecutivo podemos concluir que:

- El CRDEBJ es una organización con personalidad jurídica propia en la figura legal de Corporación de derecho público, sometida a la legislación de entidades privadas.

- Existe un sometimiento al control de las administraciones públicas en una relación que podría definirse como de dependencia indirecta: El CRDEBJ se somete a la legislación internacional, comunitaria, nacional y autonómica en materia de indicaciones geográficas, pero, al mismo tiempo, es un órgano autónomo que se autorregula con un Reglamento aprobado por el propio CRDEBJ.

- Se da una confianza entre productores y públicos del entorno del CRDEBJ.

- La adopción de una DE y, consecuentemente del CRDEBJ, ayuda al Brandy de Jerez a posicionarse de forma positiva en la sociedad de su entorno, responde el entrevistado de forma positiva en relación al posicionamiento y prestigio del BJ como producto genérico por su calidad y personalidad propias, a través de la gestión de las relaciones públicas.

- Existe una necesidad de compromiso de todas las bodegas para acometer acciones conjuntas en este sentido.

- Una indicación geográfica no se justifica de forma exclusiva desde las relaciones públicas, sino en aras de garantizar una calidad del producto. 
- Existe necesidad de aumentar la inversión del CRDEBJ en relaciones pública frente a otras acciones comunicativas.

- Las memorias tienen una rígida estructura alcanzada desde los primeros ejemplares hasta los últimos correspondientes al período analizado. Fundamentalmente, desde la memoria de 1989 hasta 2008.

- Se observa la evolución en el diseño, cuidado estético y acabado final: Las primeras memorias apenas llevan un encabezado con el logotipo... mientras que conforme avanzamos en el tiempo se evidencia la correspondiente mejora de calidad que se refleja en las impresiones, uso de logotipos y papel de mayor calidad, uso del color, maquetación...

- El área de comunicación que aumenta con los años.

\section{Conclusiones. El valor comunicativo de las indicaciones geográficas protegidas}

Desde esta perspectiva, resulta fácil entender la necesidad comunicativa que ostentan los consejos reguladores que tutelan las diferentes indicaciones geográficas (Indicación Geográfica Protegida o Denominación de Origen Protegida). $\mathrm{Y}$ es que el surgimiento de tempranas fórmulas de protección jurídico-legal de aquellos productos diferenciados por una calidad ligada a su origen geográfico, el profuso desarrollo de estas normativas en el ámbito internacional, comunitario y español, así como el creciente número de indicaciones geográficas que se ha registrado en España en los últimos años, hace reflexionar sobre la funcionalidad de las indicaciones geográficas protegidas.

A la luz de lo estudiado, las IGP tienen entre sus funciones la generación de flujos de comunicación constante entre las organizaciones y sus públicos, suponiendo una base fundamental de la gestión estratégica de la organización, esto es: desde una perspectiva directiva.

Tal y como indicábamos anteriormente, la IGP se acerca al concepto de bidireccionalidad de las Relaciones Públicas propuesto por Bernays (1998), Seitel (2002) y Lloyd y Lloyd (1990) quienes afirman el carácter bidireccional de las RR.PP., dada su vocación de hacer que el público entienda a las empresas y éstas a sus públicos, caracterizando así a los profesionales como intérpretes (Seitel, 2002:14). Sostienen Lloyd y Lloyd, además, que realizan esta labor bajo un plan determinado de acciones. En este sentido, se asemejan a los modelos cuarto y quinto de Relaciones Públicas de Grunig: doble flujo simétrico, en el que las RR.PP. tienen como función mediar entre las organizaciones y los públicos (Xifra, 2003: 53; Grunig y Hunt, 2000: 72); y antagonista cooperante, modelo en el que las Relaciones Públicas buscan y realizan pactos necesarios para, respetando las discrepancias, centrarse en las coincidencias (Pulido, 2011: 213). Para Otero (2009: 28) el modelo antagonista cooperante supone el ideal para las relaciones públicas del siglo XXI, puesto que dota a los profesionales de capacidad gestora y negociadora, y esto supone un gran valor para las organizaciones. 
Esta reflexión conlleva a determinar que el valor añadido que aportan las indicaciones geográficas es tal que merecen, en función de la reputación del producto, una protección jurídica específica a nivel internacional, comunitario y nacional.

El resultado del análisis de las legislaciones descritas en el apartado metodológico, establecido en función de los objetivos de la investigación, permite afirmar que el interés de las indicaciones geográficas gira en torno al valor que aportan, fundamentalmente, en torno a la calidad, asociación a los valores sociales y culturales de la zona de origen (que se asumen como positivos en relación a la materia prima y al saber hacer en la elaboración y producción del producto), la defensa ante el fraude y el desarrollo de económico de la zona (Martín Cerdeño, 2006; Barco Royo, 2007; Martínez Ruiz y Jiménez Arco (2006: 13-15).

Las indicaciones geográficas protegidas se relacionan con la percepción pública e imagen (positiva) del producto y su zona de origen (Jiménez Zarco, 2002; Yagüe y Jiménez, 2002a y 2002b). En este sentido, desde la perspectiva de la comunicación, estos valores positivos que se vinculan a un determinado producto por su origen geográfico, se erigen como una estrategia legítima de comunicación, que permite canalizar los mensajes que el sector productivo del producto al que ampara la indicación geográfica protegida en torno a la percepción de la calidad del producto.

De este modo, la adopción de una indicación geográfica protegida por parte de un sector productivo determinado sirve como estrategia de comunicación que le permite gestionar las relaciones de este sector productivo con los públicos de su entorno, buscando lanzar un mensaje de legitimidad y prestigio, ligada a la calidad asociada al producto por su origen geográfico.

Larissa Grunig, Grunig y Ehling (Arceo Vacas, 2004:83-86) vinculan las relaciones públicas efectivas a la calidad y a las relaciones a largo plazo con los públicos estratégicos. Y establecen una serie de indicadores a largo plazo de las relaciones entre las organizaciones y sus públicos: Reciprocidad, confianza, credibilidad, legitimidad mutua, franqueza, satisfacción y entendimiento mutuos.

Según todo lo expuesto, y a modo de conclusión final, resulta fácil entender que la adopción de fórmulas de protección que amparan determinadas indicaciones geográficas permite a los sectores productivos afectados asociarse en torno a una organización representativa de carácter semipúblico que, como tal organización, tiene unas necesidades comunicativas de gestión de sus relaciones con el entorno en el que se encuentran insertas.

\section{Bibliografía}

AA.VV. (1989). Técnicas de investigación en ciencias sociales. Datos. Observación. Entrevista. Cuestionario. Madrid: Narcea, S.A. de ediciones.

AA.VV. (1995). Historia y cultura del vino en Andalucía. Sevilla, Juan José Iglesias Rodríguez (ed.) Secretariado de Publicaciones de la Universidad de Sevilla. 
AA.VV. (1995). La comunicación en la empresa. Madrid: DIRCOM.

AA.VV. (1997). El libro práctico de la comunicación y las relaciones públicas. Madrid: Ed. Folio.

AA.VV. (2003). Derechos intelectuales. Ciudad de Buenos Aires: Editorial Astrea de Alfredo y Ricardo Depalma.

AA.VV. (1992). Diccionario de la Lengua Española. Real Academia Española. Madrid: Espasa Calpe.

AA.VV. (2001). Diccionario de la Lengua Española. Real Academia Española. Madrid: Espasa Calpe.

AA.VV. (2002). El libro del Brandy de Jerez. Madrid: Consejo Regulador del Brandy de Jerez.

Ander-Egg, E. (1995). Técnicas de investigación social. Buenos Aires: Lumen.

Arceo Vacas, J.L. (1988). Fundamentos para la teoría y la técnica de las relaciones públicas. Barcelona: P.P.U.

Arceo Vacas, J.L. (2004). Las relaciones públicas en España. Madrid: Mc Graw Hill.

Barco, E. (2007a). Denominaciones de origen. La incidencia de la localización y deslocalización. En: Distribución y consumo, 96. Madrid: Mercasa. Recuperado de: http://www.mercasa.es/files/multimedios/1288205986_ DYC_2007_96_27_39.pdf [Consultado el 14-10-2008].

Barco, E. (2007b). La reforma de la OCM del sector vitivinícola. En: Agricultura familiar en España. Unión de Pequeños Agricultores y Ganaderos. Recuperado de: http://www.upa.es/anuario_2007/pag_096-111_barco.pdf. [Consultado el 14-10-2008].

Bardin, L. (1986). Análisis de contenido. Madrid: Akal Universitaria.

Berenguer G., L. (2006) El registro de indicaciones geográficas de terceros Estados en la Unión Europea. En: Simposio Internacional de Indicaciones Geográficas. Comunidad Andina de Naciones, Lima, 11 y 12 de enero del 2006.

Bello, L., Gómez, J. (1996). Las denominaciones de origen y otras señas de calidad en las estrategias de diferenciación de los productos agroalimentarios: una propuesta metodológica. En: Cuadernos aragoneses de economía, vol. 6, 2. Zaragoza: Facultad de Ciencias Económicas y Empresariales de la Universidad de Zaragoza.

Bernays, E. L. (1998). Cristalizando la opinión pública. Barcelona: Gestión 2000 .

Black, S. (2001). ABC de las relaciones públicas. Barcelona: Ed. Gestión 2000. 
Bonilla, C. (1988). La comunicación función básica de las relaciones públicas. México: Ed. Trillas.

Botan, C. y Hazleton, V. (2006). Public Relations Theory II. Mahwah, New Jersey: Lawrence Earlbaum Associates, Inc.

Castillo, A. (2010). Introducción a las relaciones públicas. Málaga: IIRP.

Cutlip y Center (2001). Relaciones públicas eficaces. Barcelona: Gestión 2000.

Del Rey, R. (2006). El vino español y el mundo del vino. Estrategias de comercialización. En: El mundo del vino, 2005. Madrid: FEV.

De Soussa Borda, A. L. (2003). Indicaciones geográficas. En: Derechos intelectuales. Buenos Aires: Editorial Astrea de Alfredo y Ricardo Depalma.

Duverger, M. (1996). Métodos de las Ciencias Sociales. Barcelona: Ariel Sociología.

Fernández De Bobadilla, V. (1990). El Brandy de Jerez. Madrid: Creaciones y Servicios Editoriales, S.A.

Galdós Urrutia, R. (2004). La intervención pública en la promoción de la calidad agroalimentaria: normativa comunitaria, española y vasca. En: Investigaciones geográficas, 34. Alicante: Instituto Universitario de Geografía. Universidad de Alicante.

Gastiaburu, K. (2008). Indicaciones geográficas y denominaciones de origen: modos de protección internacional de los productos de calidad agroalimentarios, vitivinícolas, artesanales y otros. En: Revista de la Competencia y la Propiedad Intelectual, 7. Lima: Instituto Nacional de Defensa de la Competencia y de la Propiedad Intelectual. Recuperado de: http://aplicaciones.indecopi.gob. pe/ArchivosPortal/boletines/recompi/castellano/articulos/primavera2008/04Ind icacionesGeograficas.pdf. [Consultado el 22-04-2009].

Gómez Díaz, A. M. (2002). La manzanilla. Historia y cultura. Las bodegas de Sanlúcar. Sanlúcar de Barrameda: Pequeñas ideas.

Gómez Lozano, M. M. (2004). Denominaciones de origen y otras indicaciones geográficas. Navarra: Aranzadi.

González Botija, F. (2003). El régimen jurídico de los vinos de calidad producidos en región determinada. Barcelona: Ed. Atelier.

Grunig, J. E. y Hunt, T. (2000). Dirección de relaciones públicas, Barcelona: Gestión 2000.

Jiménez Blanco, P. (1996). Las denominaciones de origen en el derecho de comercio internacional. Madrid: Editorial Eurolex.

Jiménez Zarco, A.I. (2002). La percepción de las denominaciones de origen Mancha y Valdepeñas de España. En: Agroalimentaria, vol. 8, 14. Mérida: Cen- 
tro de Investigaciones Agroalimentarias. Recuperado de: http://www.saber.ula. ve/bitstream/123456789/17820/1/articulo14_4.pdf. [Consultado el 20-10-2009].

Jiménez Zarco, A.I. y Gómez, M.A. (1997). La denominación de origen como marca: la procedencia como elemento de identidad. En: Investigación y marketing, 55. Barcelona: Asociación Española de Estudios de Mercado, Marketing y Opinión.

Krippendorf, K. (1990). Metodología del análisis de contenido. Teoría y práctica. Barcelona: Paidós Comunicación.

Ledingham, J.A. (2006). Relationship management: a general theory of public relations. En: Public Relations Theory II. Mahwah, New Jersey: Lawrence Earlbaum Associates, Inc.

L’Etang, J. y Pieczka, M. (2006). Public Relations. Critical debates and contemporary practice. Mahwah, New Jersey: Lawrence Earlbaum Associates, Inc.

López Benítez, M. (1996). Las denominaciones de origen. Barcelona: Editorial Cedecs.

López Benítez, M. (2004). Del estatuto del vino a las leyes del vino: un panorama actual y de futuro de la ordenación vitivinícola en España. Madrid: Editorial Cívitas.

Maroño Gargallo, Ma del Mar (2002). La protección jurídica de las denominaciones de origen en los derechos español y comunitario, Madrid: Editorial Marcial Pons.

Martín Cerdeño, V. J. (2006). Denominaciones de origen: una revisión en el mercado español. En: Distribución y consumo, 90. Madrid: Mercasa. Recuperado de: http://www.mapa.es/ministerio/pags/biblioteca/revistas/pdf_DYC/ DYC_2006_90_87_113.pdf. [Consultado el 05-11-2009].

Martínez Ruiz, M.P. Y Jiménez Zarco, A.I. (2006). La potenciación del origen en las estrategias de marketing de productos agroalimentarios. Objetivos, situación e implicaciones. En: Boletín económico del ICE, 2880. Madrid. Recuperado de: http://www.revistasice.com/CachePDF/BICE_2880_1330_A7AB062C8AB61FC37C68753B1DEF0DB.pdf [Consultado el 10-12-2009].

Nahoum, Ch. (1990). El proceso de la entrevista. Editorial Kapelusz, México.

Neumayer, E. y Perkins, R. (2005). Uneven geographies of organizational practice: explanning the cross - national transfer and adoption os ISO 9000. En: Economic Geography, 81 (3), pp. 237-259.

Noguero, A. (1995). La función social de las relaciones públicas: historia, teoría y marco legal. Barcelona: ESRP.

Otero Alvarado, M. T. (2000). Teoría y estructura del ceremonial y el protocolo, Sevilla: Mergablum edición y comunicación. 208 
Otero Alvarado, M. T. (2009). Protocolo y organización de eventos. Barcelona: Ed. UOC.

Otero Alvarado, M. T. (1996). Un Toro negro y enorme: del diseño publicitario industrial a las relaciones públicas Institucionales. Algunas consideraciones sobre la comunicación empresarial e institucional. En: Questiones Publicitarias, Sevilla - Métodos, Análisis y Estrategias de la Comunicación Empresarial e Institucional, 1.

Pérez-Tenessa, A. (2000). El vino y su régimen jurídico. Madrid: MAPA.

Peñín, J. (2008). Historia del vino. Madrid: Espasa Calpe.

Sainz, H. (2000). Actitud de los consumidores ante los vinos con denominación de origen. En: Distribución y consumo, 55. Madrid: Mercasa. Recuperado de: http://www.mapa.es/ministerio/pags/biblioteca/revistas/pdf_DYC/ DYC_2000_55_131_143.pdf. [Consultado el 04-09-2009].

Schiavone, E. (2003). Indicaciones geográficas. Derechos intelectuales. Buenos Aires: Editorial Astrea de Alfredo y Ricardo Depalma.

Seitel, F. (2002). Teoría y práctica de las relaciones públicas. Madrid: Prentice Hall.

Sierra, F. (1998). Función y sentido de la entrevista cualitativa en investigación social. En: Galindo, J. (1998). Técnicas de investigación en sociedad, cultura y comunicación. México: Pearson.

Wilcox, D. L., Cameron, G.T. y Xifra, J. (2009). Relaciones públicas. Estrategias y tácticas. Madrid: Pearson Addison Wesley.

Yagüe, M.J. y Jiménez, A.I. (2002a). Estrategias de diferenciación en los mercados agroalimentarios: la percepción de la imagen de la Denominación de Origen. En: Investigación y Marketing, 76. Barcelona: Asociación Española de Estudios de Mercado, Marketing y Opinión.

Yagüe, M.J. y Jiménez, A.I. (2002b). La Denominación de Origen en el desarrollo de estrategias de diferenciación: percepción y facetas de su utilización en las sociedades vinícolas de La Mancha y Valdepeñas. En: Revista Española de Estudios Agrosociales y Pesqueros, 197. Madrid: Ministerio de Agricultura, Pesca y Alimentación.

Xifra, J. (2003). Teoría y estructura de las relaciones públicas. Madrid: Mc Graw Hill.

- (2005). Planificación estratégica de relaciones públicas. Barcelona: Paidós.

- (2007). Técnicas de las Relaciones Públicas. Barcelona: Editorial UOC. 


\section{Referencia de este artículo}

Pulido, Marta; Jiménez, Gloria y Silva Robles, Carmen (2012). Label de calidad e indicaciones geográficas protegidas como estrategia empresarial: El caso Brandy de Jerez desde una perspectiva de las RR.PP. En: adComunica. Revista Científica de Estrategias, Tendencias e Innovación en Comunicación, $\mathrm{n}^{\circ} 4$. Castellón: Asociación para el Desarrollo de la Comunicación adComunica, Universidad Complutense de Madrid y Universitat Jaume I, 171-198. DOI: http://dx.doi. org/10.6035/2174-0992.2012.4.11 